\title{
Article \\ BiFC Method Based on Intraorganellar Protein Crowding Detects Oleate-Dependent Peroxisomal Targeting of Pichia pastoris Malate Dehydrogenase
}

\author{
Jean-Claude Farré *(D, Paul Li and Suresh Subramani * \\ Section of Molecular Biology, Division of Biological Sciences, University of California, \\ San Diego, CA 92093-0322, USA; py1001@ucsd.edu \\ * Correspondence: jfarre@ucsd.edu (J.-C.F.); ssubramani@ucsd.edu (S.S.)
}

check for

updates

Citation: Farré, J.-C.; Li, P.;

Subramani, S. BiFC Method Based on Intraorganellar Protein Crowding

Detects Oleate-Dependent

Peroxisomal Targeting of

Pichia pastoris Malate Dehydrogenase.

Int. J. Mol. Sci. 2021, 22, 4890.

https://doi.org/10.3390/

ijms22094890

Academic Editor: Atsushi Matsuzawa

Received: 16 March 2021

Accepted: 29 April 2021

Published: 5 May 2021

Publisher's Note: MDPI stays neutral with regard to jurisdictional claims in published maps and institutional affiliations.

Copyright: (c) 2021 by the authors. Licensee MDPI, Basel, Switzerland. This article is an open access article distributed under the terms and conditions of the Creative Commons Attribution (CC BY) license (https:// creativecommons.org/licenses/by/ $4.0 /)$.

\begin{abstract}
The maintenance of intracellular NAD ${ }^{+} / \mathrm{NADH}$ homeostasis across multiple, subcellular compartments requires the presence of $\mathrm{NADH}$-shuttling proteins, which circumvent the lack of permeability of organelle membranes to these cofactors. Very little is known regarding these proteins in the methylotrophic yeast, Pichia pastoris. During the study of the subcellular locations of these shuttling proteins, which often have dual subcellular locations, it became necessary to develop new ways to detect the weak peroxisomal locations of some of these proteins. We have developed a novel variation of the traditional Bimolecular Fluorescence Complementation (BiFC), called divergent BiFC, to detect intraorganellar colocalization of two noninteracting proteins based on their proximity-based protein crowding within a small subcellular compartment, rather than on the traditional proteinprotein interactions expected for BiFC. This method is used to demonstrate the partially peroxisomal location of one such $P$. pastoris NADH-shuttling protein, malate dehydrogenase B, only when cells are grown in oleate, but not when grown in methanol or glucose. We discuss the mode of NADH shuttling in P. pastoris and the physiological basis of the medium-dependent compartmentalization of $P p \mathrm{MdhB}$.
\end{abstract}

Keywords: redox balance; NADH shuttle; peroxisomal malate dehydrogenase; environment-dependent peroxisomal targeting; intraorganellar protein crowding

\section{Introduction}

The Bimolecular Fluorescence Complementation (BiFC) assay is a protein proximitybased method developed initially to directly visualize protein-protein interaction in vivo using live-cell imaging or fixed cells. BiFC was first developed using two nonfluorescent protein fragments of the enhanced yellow fluorescent protein (EYFP), which is an Aequorea victoria GFP variant protein [1]. The YFP was later improved by introducing mutations to reduce its sensitivity to $\mathrm{pH}$ and chloride ions, thus generating a super-enhanced YFP, named Venus fluorescent protein, without reduction in fluorescence at $37{ }^{\circ} \mathrm{C}$, as often observed with EYFP [2]. The fluorescence signal is regenerated by complementing two nonfluorescent protein fragments (e.g., the Venus N-terminal 1-158 amino acids, called VN, and its C-terminal 159-239 amino acids, named VC) that are brought together by the interaction between their respective fusion partners.

Here we use BiFC somewhat differently, in a method dubbed "divergent BiFC", to detect colocalization of two noninteracting proteins fused to $\mathrm{VN}$ and $\mathrm{VC}$, respectively, within a restricted subcellular compartment, the peroxisome. We show that fluorescence can be generated by proximity-based protein crowding in peroxisomes.

We used the divergent BiFC method to understand the NADH-shuttling proteins that are normally localized to three subcellular compartments, namely, the mitochondria, peroxisomes, and the cytosol [3]. Mechanisms for the maintenance of the $\mathrm{NAD}^{+} / \mathrm{NADH}^{2}$ 
redox balance are conserved in evolution, and the sum of the $\mathrm{NAD}^{+}$and NADH concentration is around $1 \mathrm{mM}$ in Saccharomyces [4]. This homeostasis requires that as $\mathrm{NAD}^{+}$is reduced, NADH must be oxidized intracellularly. The pathways by which this homeostasis is achieved have been reviewed elsewhere [4]. What is important to note, however, is that NADH metabolism occurs in different subcellular compartments, whose membranes are not permeable to these cofactors. To circumvent this challenge, NADH-shuttling proteins exist in eukaryotic cells that facilitate the efficient transfer between subcellular compartments, such as the mitochondria, where NADH is used to generate ATP, peroxisomes where NADH is generated in yeast cells grown on fatty acids as the sole carbon source and the cytosol $[4,5]$. In the methylotrophic yeast, P. pastoris, none of the shuttling mechanisms that transport NADH have been described, and the proteins responsible have not been identified.

We demonstrate the dual localization to mitochondria and the cytosol for two such Pichia pastoris NADH-shuttling proteins, glycerol-3-phosphate dehydrogenase ( $P p \mathrm{GpdA})$ and one isoform of malate dehydrogenase $\mathrm{A}(P p \mathrm{MdhA})$ to both mitochondria and cytosol. Additionally, using the divergent BiFC method, we detected the peroxisomal and cytosolic localization of another NADH-shuttling protein, malate dehydrogenase B ( $P p \mathrm{MdhB})$. We discuss the physiological role of the peroxisomal localization of this particular malate dehydrogenase isoform (PpMdhB) only in oleate-, but not in methanol- or glucose-grown cells.

\section{Results}

\subsection{NADH-Shuttling Proteins in P. pastoris}

S. cerevisiae encodes three malate dehydrogenases (Mdh: Mdh1, Mdh2, and Mdh3) that display strong sequence similarities between them, the most significant of which are the presence of either a mitochondrial or a peroxisomal targeting signal (PTS) (Figure S1A). Similarly, the two glycerol-3-phosphate dehydrogenases (Gpd: Gpd1 and Gpd2) share a strong protein sequence homology, but one of them has a PTS (Figure S1B).

To identify the $P$. pastoris counterpart of these dehydrogenases, we searched the P. pastoris GS115 database performing a protein-protein BLAST (BLASTP) using the three isoforms of Mdh and two isoforms of Gpd from $S$. cerevisiae as a query. We found that P. pastoris has two isoforms of Mdh and only one isoform of Gpd (Figure S1A,B). Since we did not know which isoforms in P. pastoris correspond to those in S. cerevisiae, we refer to them as $P p \mathrm{MdhA}, P p \mathrm{MdhB}$, and $P p \mathrm{GpdA}$.

We analyzed the $P$. pastoris NADH-shuttling proteins sequences manually and using the prediction algorithm of the PTS1 predictor version 1 (http:/ / mendel.imp.ac.at/pts1/ accessed on 14 March 2021), but no obvious SKL tripeptide, or its variant, was found their C-termini (Figure S1 and Table 1). A PTS score equal to or greater than 0 would potentially have a predicted PTS1, while a PTS score under -10 would not be predicted to be a PTS1 sequence [6]. Based on this scoring system, all of the Mdh and Gpd proteins listed for P. pastoris and S. cerevisiae scored below -10 and did not have a predicted PTS1, except for ScMdh3 that has a PTS score above 0 , as well as an SKL as the PTS1 at its C-terminus (Table 1).

When analyzing the N-terminus of the NADH-shuttling protein homologs, no sequences corresponding to the PTS2 consensus [7], (R/K)-(L/V/I)-(X) $)_{5}(\mathrm{H} / \mathrm{Q})-(\mathrm{L} / \mathrm{A})$ or (R/K)-(L/V/I/Q)-X-X-(L/V/I/H/Q)-(L/S/G/A/K)-X-(H/Q)-(L/A/F), were found in the P. pastoris Mdh or Gpd proteins using EMBOSS fuzzpro that can search for the different combinations of the consensus sequences [8]. Only ScGpd1 has a PTS2 at the N-terminus with the consensus sequence (RLNLTSGHL) (Figure S1B). The closest PTS2 consensus near the N-terminus of the $P$. pastoris proteins had a match of only 6 out of 9 amino acids for Pp GpdA, PpMdhA, and PpMdhB. 
Table 1. In silico analysis of malate and glycerol 3-phosphate dehydrogenases in P. pastoris and S. cerevisiae. PTS1 scores were based on the C-terminal sequences of the dehydrogenases. Scores greater than 0 typically have a predictable PTS1. MitoProt scores were based on the N-terminal predictions for mitochondrial presequences. MitoProtII scores with a probability greater than 0.5 are more likely to be mitochondrial proteins.

\begin{tabular}{ccccc}
\hline Protein & C-Terminal & PTS1 Score & N-Terminal & MitoProt Score \\
\hline$S c$ Mdh1 & NIEKGVNFVASK & -48.5 & MLSRVAKRAFSSTVANP & 0.9910 \\
$S c$ Mdh2 & GLEFVASRSASS & -38.2 & Not predictable & 0.0376 \\
$S c$ Mdh3 & KGKSFILDSSKL & 4.9 & Not predictable & 0.1859 \\
$P p$ MdhA & IAKGQEFVKQNP & -37.7 & MLSTIAKRQFSSSASTA & 0.9549 \\
$P p$ MdhB & NIAKGTAFIAGN & -52.6 & Not predictable & 0.1410 \\
$S c G p d 1$ & PDMIEELDLHED & -101.6 & MSAAADRLNLTSGHLNAGRKRS & 0.3132 \\
$S c G p d 2$ & PEMIEELDIDDE & -86.8 & MLAVRRLTRYTFLKRTH & 0.9932 \\
$P p G p d A$ & FNKTEDVKHWED & -58.1 & MYLTSTVRALPVHFFRSRHCIRT & 0.6471 \\
\hline
\end{tabular}

However, $P p \mathrm{MdhA}$ and $P p \mathrm{GpdA}$ have a high probability (above the threshold score of $0.5)$ of being targeted to the mitochondria and contain an identifiable mitochondrial presequence at the N-terminus based on the MitoProtII program (https: / /bio.tools/MITOPROT_II accessed on 14 March 2021) [9]. More specifically, PpMdhA has a probability of 0.948 and an 18 amino acid, mitochondrial presequence (MLSTIAKRQFSSSASTA), while PpGpdA has a probability of 0.6471 for being translocated to the mitochondria with a 24 amino acid, mitochondrial presequence (MYLTSTVRALPVHFFRSRHCIRT) (Table 1). ScMdh1 and $S c G p d 2$ have a probability greater than 0.9 and are known to have a mitochondrial localization, which is similar to $P p \mathrm{MdhA}$ that also has a high probability for mitochondrial targeting. $P p$ GpdA has a lower probability than the S. cerevisiae mitochondrial NADHshuttling proteins (Mdh1 and Gpd2), but a higher probability than cytosolic or peroxisomal shuttling dehydrogenases (ScMdh2, ScMdh3, and $S c G p d 1$ ). In conclusion, $P p$ MdhA has a high chance of being a mitochondrial enzyme and could be the homolog of ScMdh1. $P p \mathrm{MdhB}$ does not have a distinct targeting signal, so the putative localization cannot be elucidated in silico.

\subsection{Localization of PpMdhA-GFP, PpMdhB-GFP, and PpGpdA-GFP in Different Carbon Sources}

We found $P p \mathrm{MdhA}$ and $P p \mathrm{GpdA}$, both lacking an identifiable PTS1 and PTS2 sequence, to have a high likelihood of mitochondrial targeting in silico. Of the three dehydrogenases, $P p \mathrm{MdhB}$ has the lowest probability for mitochondrial targeting, hence the best candidate to be at least partially localized to peroxisomes. In S. cerevisiae, Mdh3 is important for intraperoxisomal redox balance of $\mathrm{NAD}(\mathrm{H})$ in oleate, but not in other media. If P. pastoris shares a similar shuttling mechanism with $S$. cerevisiae for transporting NADH from the peroxisome to the mitochondria and maintaining the intraperoxisomal redox balance in oleate, then it would be important to study the localization of $P p \mathrm{MdhA}, P p \mathrm{MdhB}$, and $P p \mathrm{GpdA}$ in glucose, methanol, and oleate.

To localize the NADH-shuttling proteins in P. pastoris, we fused GFP to the C-terminus of $P p \mathrm{MdhA} / \mathrm{B}$ and $P p \mathrm{GpdA}$, and expressed these GFP fusion proteins from their endogenous promoters in wild-type cells (Figure 1). We also transformed a mitochondrial and peroxisomal marker, Tom20-mRFP and BFP-SKL, respectively, into the wild-type strains containing the shuttling dehydrogenase-GFP fusion proteins to determine if there was colocalization at the mitochondria or peroxisomes. Both PpMdhA-GFP and P $p$ GpdA-GFP colocalized with the mitochondrial marker in glucose, methanol, and oleate media, but also appeared to have partially cytosolic fluorescence, suggesting that these two proteins may possess dual localization in P. pastoris in all three media (Figure 1A,B). 


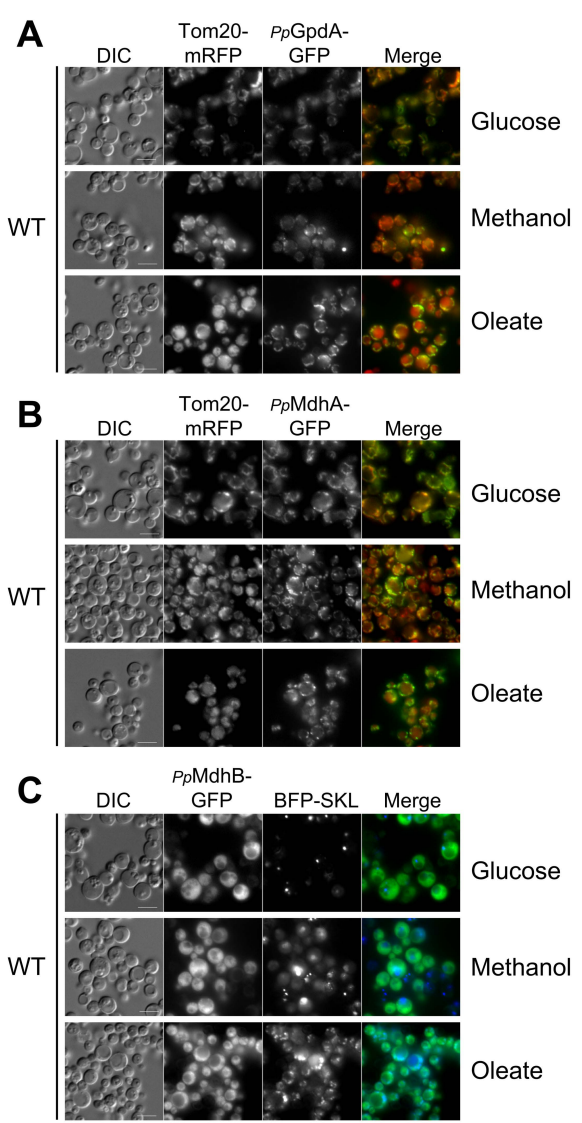

Figure 1. Localization of P. pastoris NADH-shuttling proteins fused with GFP. Fluorescence microscopy of strains grown in glucose, methanol, and oleate overnight. GFP was fused to the C-terminus of the NADH shuttling proteins, and the fusion proteins were expressed from their endogenous promoters. (A) Wild-type cells expressing PpGpdA-GFP, (B) Wild-type cells expressing PpMdhA-GFP, and (C) Wild-type cells expressing PpMdhB-GFP. Colocalization with the mitochondria and peroxisomes was observed with Tom20-mRFP or BFP-SKL as mitochondrial and peroxisomal markers, respectively. Bars: $5 \mu \mathrm{m}$.

In contrast, $P p$ MdhB-GFP differed in that it appeared cytosolic in all three media with no obvious intraorganelle localization (Figure 1C). Based on the cytosolic and mitochondrial localization, supported by its likelihood for mitochondrial targeting, $P p \mathrm{GpdA}$ is likely the homolog of $S c \mathrm{Gpd} 2$. The mitochondrial localization confirmed the high probability for mitochondrial targeting for $P p \mathrm{MdhA}$, making it likely to be the homolog of ScMdh1. Finally, based on the cytosolic localization, $P p \mathrm{MdhB}$ appeared to be like ScMdh2.

Based on the localization of NADH-shuttling proteins in all the media tested in P. pastoris, it would appear that NADH might not shuttle out of the peroxisome, as neither $P p \mathrm{Mdh}$ isoforms, nor $P p \mathrm{Gpd}$, had any detectable peroxisomal localization. However, given the importance and conservation of the NADH shuttle and peroxisomal NADH production, we sought a more sensitive technique to detect small pools of peroxisomally-localized $\mathrm{NADH}$-shuttling proteins.

\subsection{An Assay to Improve Detection of Peroxisomally-Localized Proteins}

We hypothesized that a small fraction of the NADH-shuttling proteins might localize at peroxisomes, but that this localization was probably drowned out by the predominantly cytosolic fraction. To detect a putative small peroxisomal fraction, we used the bimolecular fluorescence complementation (BiFC) technique.

BiFC uses a fluorescent Venus protein (V) that is split into two, nonfluorescent halves, called VN and VC, which are typically fused to two proteins that may physically interact or be in close proximity to bring VN and VC together to reconstitute GFP, and yield 
fluorescence [10]. For investigating the localization of the NADH-shuttling proteins, we took advantage of the latter case. We targeted the VN moiety to the peroxisome through the most efficient PTS1 pathway [11], by fusing the ePTS1 (LGRGRRSKL) to the C-terminus of VN and fusing the NADH-shuttling proteins with VC. To increase the chances that VN and VC find each other and yield fluorescence, we overexpressed both fusion proteins. The PTS1 pathway is extremely efficient, and a large fraction of VN-ePTS1 was expected to localize to peroxisomes [11]. If a small fraction of the NADH-shuttling proteins fused to $\mathrm{VC}$ is also imported to the peroxisomes, the fluorescence at the peroxisomes should exceed that in the cytosol, allowing detection by regular fluorescence microscopy (Figure 2).

A

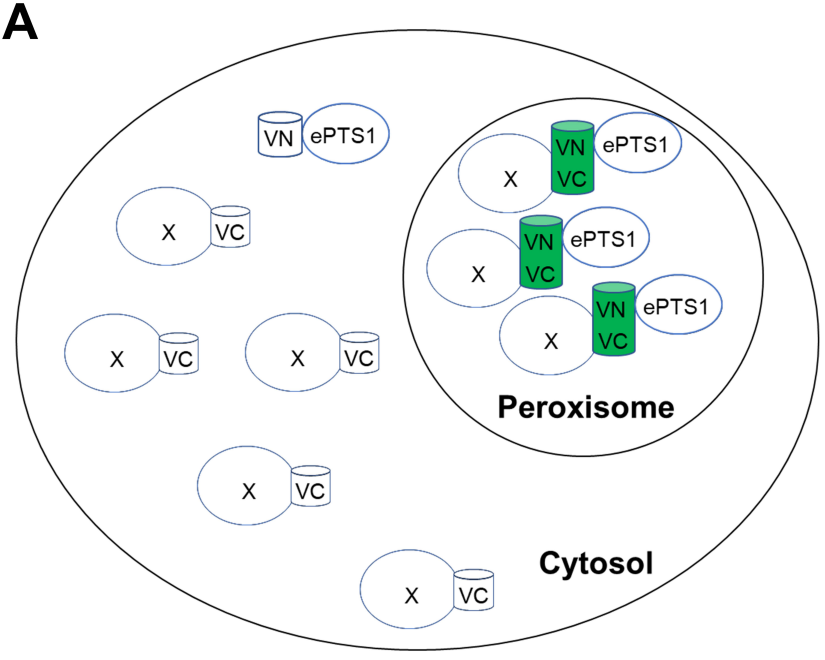

B

\begin{tabular}{lll}
\hline X-VC & VN-ePTS1 & Peroxisomal Fluorescence \\
\hline & & No \\
Cytosol & Peroxisome & Yes \\
Peroxisome & Peroxisome & No \\
Mitochondria & Peroxisome & Yes \\
Cytosol \& Peroxisome & Peroxisome & No \\
Cytosol \& Mitochondria & Peroxisome & Yes
\end{tabular}

Figure 2. Expected fluorescence using the divergent BiFC assay. (A) Schematic representation of the expected outcome of the assay. (B) Potential subcellular localizations that may yield peroxisomal fluorescence. (C) Plasmid construct (VN and VC-BiFC moieties, POT1 and AOX terminator-transcription terminator, HIS4 - histidine selection marker for yeast, $\mathrm{Amp}^{\mathrm{R}}$ —ampicillin resistance gene, HTX1 bidirectional promoter-strong constitutive bidirectional promoter, ePTS1enhanced peroxisomal-targeting signal 1, POT1—thiolase open reading frame, ORI—bacterial replication origin). AvrII and AfIII were used for double digestion to replace POT1 with the gene encoding the protein of interest.

To prove the concept of the divergent BiFC assay, we coexpressed together with VN-ePTS1, a peroxisomal noninteracting protein, thiolase (Pot1), fused with VC at its C-terminus. Thiolase is synthesized in the cytosol and imported via the PTS2 pathway into peroxisomes, where it catalyzes the last step of the $\beta$-oxidation pathway [12]. Since the PTS2 nonapeptide is near the amino terminus of Pot1, the VC moiety was fused at the C-terminus. Thus, both the Pot1-VC with its PTS2, and VN-ePTS1 with its PTS1 can still be recognized by their respective PTS receptors, Pex7 and Pex5, respectively, and imported into peroxisomes.

The overexpression and peroxisomal crowding of these noninteracting proteins was expected to bring together VN and VC to yield fluorescence. The plasmid coexpressing Pot1-VC and VN-ePTS1 was integrated into the genome of $P$. pastoris wild-type cells 
expressing peroxisomal Pex3-mRFP from its own promoter and analyzed by fluorescence microscopy after induction in glucose, methanol, or oleate media (Figure 3). P. pastoris glucose-grown cells contain a single small peroxisome (area: $\sim 0.05 \mu \mathrm{m}^{2}$ ), and when cells are grown in media requiring peroxisome metabolism, the peroxisome size and number increase [13]. The most commonly used media for peroxisome proliferation in P. pastoris are oleate and methanol. When cells are grown in oleate media overnight, peroxisome number and area increase to $\sim 6$ and to $\sim 0.08 \mu \mathrm{m}^{2}$, respectively, and peroxisomes are dispersed in the cytosol near the cell periphery. Similarly, when cells are grown overnight in methanol media, peroxisome number and area increase to $\sim 4$ and to $\sim 0.4 \mu \mathrm{m}^{2}$, respectively, and are clustered.
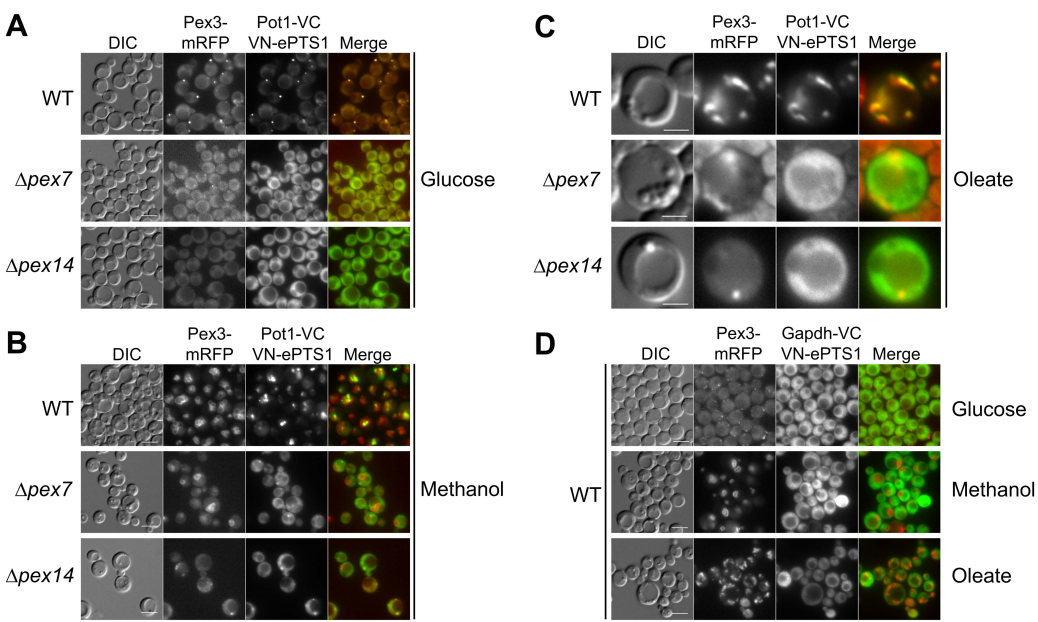

Figure 3. Feasibility of the divergent BiFC assay using known peroxisomal matrix and cytosolic proteins. The PTS2-containing protein, thiolase (Pot1), was fused to VC (Pot1-VC), and the PTS1 was fused to VN (VN-ePTS1). Strains were grown overnight in either (A) glucose, (B) methanol, or (C) oleate. (D) Strains were grown overnight in glucose, methanol, and oleate, and peroxisomal fluorescence were not observed for a cytosolic protein (Gapdh). Peroxisomes were visualized using Pex3-mRFP. Bars for (A,B,D) $5 \mu \mathrm{m}$; bars for (C) $2 \mu \mathrm{m}$.

As hypothesized, the overexpression of the two peroxisomal chimera proteins Pot1VC and VN-ePTS1 in wild-type cells, showed a strong fluorescence at the peroxisome in every medium, confirmed by the colocalization with Pex3-mRFP, indicating the two nonfluorescent halves of the Venus proteins were reconstituted in the peroxisomes, most likely due to their proximity created by their high abundance in a confined area (Figure 3A-C).

To confirm that the peroxisomal fluorescence observed in our divergent BiFC assay was a consequence of the Venus reconstitution in the peroxisomal matrix and not a weak fluorescence of the Venus moiety itself, we knocked out different essential components of peroxisomal matrix protein import. With this purpose, we transformed the plasmid coexpressing Pot1-VC and VN-ePTS1, along with Pex3-mRFP to mark peroxisomes, into cells mutated for the PTS2 receptor $(\Delta p e x 7)$ and the peroxisomal receptor docking protein ( $\Delta$ pex14) and tested them in glucose, methanol, and oleate (Figure 3).

When we analyzed cells lacking the PTS2 receptor in the three media, no fluorescence was observed at the peroxisome, although we noticed some cytosolic fluorescence (Figure 3A-C). These results were expected, at least for the lack of peroxisomal fluorescence, as Pot1 was not expected to be imported into the peroxisome without its PTS2 receptor, Pex7. However, the cytosolic fluorescence was unexpected because VN-ePTS1 should be imported into the peroxisome in the presence of the PTS1 receptor, Pex5, which might suggest that a fair amount of the VN-ePTS1 synthesized in the cytosol remained there.

When we analyzed cells lacking the peroxisomal receptor docking protein Pex14, no peroxisomal fluorescence was observed in any media as expected (Figure $3 \mathrm{~A}-\mathrm{C}$ ), because, in the absence of Pex14, neither Pex5 nor Pex7 would be expected to dock at the peroxisomal 
membrane to import their respective cargoes. Similar to the result in the $\Delta p e x 7$ cells, a cytosolic fluorescence was observed in the $\Delta$ pex 14 cells, although in this case, the result was highly expected as both VN-ePTS1 and Pot1-VC were not supposed to be imported. In addition, the fluorescence in the cytosol indicated the constitutive HTX1 promoter is strong enough to crowd even the cytosol with the two Venus moieties.

To verify the specificity of the divergent BiFC assay, we coexpressed GAPDH-VC and VN-ePTS1 in wild-type cells and observed that in all three media, the fluorescence was localized in the cytosol, and no colocalization with Pex3-mRFP was observed (Figure 3D). To verify that Gapdh-VC was capable of being transported to, and interacting with VNePTS1 in, the peroxisomes, we appended a PTS1 at its C-terminus (Gapdh-VC-SKL) and observed a clear peroxisomal signal (Figure S2). This control proves that the lack of peroxisomal fluorescence of Gapdh-VC is because this protein does not piggyback into peroxisomes with VN-ePTS1.

Thus, the divergent $\mathrm{BiFC}$ assay worked as expected with a strong peroxisomal fluorescence for Pot1, which could be abolished by the deletion of PEX7 and PEX14 genes, and no peroxisomal fluorescence was observed when we swapped Pot1 with the cytosolic protein Gapdh in wild-type cells.

\subsection{Testing the Localization of the NADH-Shuttling Proteins Using the BiFC Assay}

We then tested the localization of our NADH-shuttling proteins by transforming $P p$ GpdA-, $P p$ MdhA-, or P $p$ MdhB-VC and VN-ePTS1 plasmids, along with those expressing peroxisomal markers into wild-type cells. These fusions with VC at the C-terminus of the $\mathrm{NADH}$-shuttling proteins would test whether these proteins had either an $\mathrm{N}$-terminal or internal PTS, while also potentially blocking peroxisomal import by a C-terminal PTS.

In wild-type cells, we observed mitochondrial fluorescence for $P p \mathrm{GpdA}$ and $P p \mathrm{MdhA}$ in glucose, methanol, and oleate (Figure 4A,B). Peroxisomal fluorescence was not observed, suggesting no colocalization with Pex3-mRFP, as expected if VN-ePTS1 was imported into the peroxisome and $P p \mathrm{GpdA}$ or $P p \mathrm{MdhA}$ was imported to the mitochondria, as predicted by our sequence analysis.

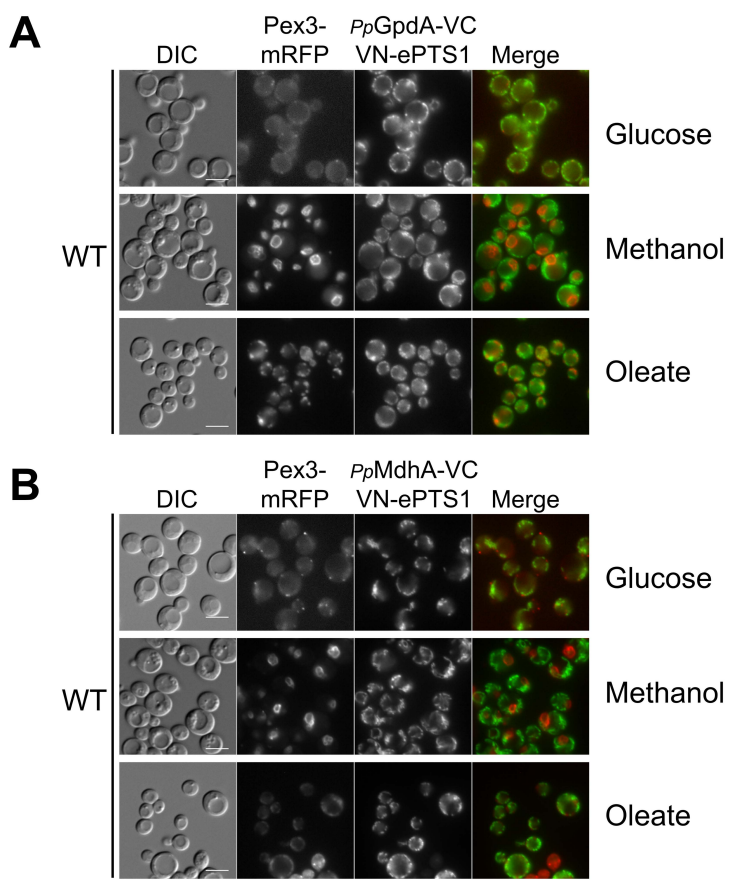

Figure 4. Localization of $P p \mathrm{GpdA}$ and $P p \mathrm{MdhA}$ in wild-type cells using the divergent BiFC assay. Strains were grown in glucose, methanol, and oleate overnight. Peroxisomes were visualized using Pex3-mRFP. (A) PpGpdA-VC; (B) PpMdhA-VC. Bars: $5 \mu \mathrm{m}$. 
However, we did not expect detectable mitochondrial fluorescence, unless overexpressed VN-ePTS1 was somehow mislocalized (partially) to mitochondria. A possible explanation would be that due to the overexpression, sufficient amounts of the $P p \mathrm{Gpd}-\mathrm{VC}$ or PpMdhA-VC fusions come in contact with VN-ePTS1 in the cytosol, yielding fluorescence, and due to the irreversible nature of the BiFC interaction get imported to, or accumulated at, the mitochondrial outer membrane.

More importantly, in wild-type cells, $P p \mathrm{MdhB}$ displayed cytosolic fluorescence in glucose and methanol, but in oleate, it was partially peroxisomal and cytosolic (Figure 5A). Similar to Gapdh, $P p$ MdhB exhibited cytosolic fluorescence, as $P p$ MdhB remained mostly in the cytosol and reconstituted the Venus protein with a portion of VN-ePTS1 synthesized in the cytosol, indicating that $P p \mathrm{MdhB}$ is a cytosolic protein. In oleate, $P p \mathrm{MdhB}$ displayed cytosolic and peroxisomal fluorescence colocalized with Pex3-mRFP, suggesting that $P p \mathrm{MdhB}$ has a dual localization in the cytosol and peroxisomes in this medium.

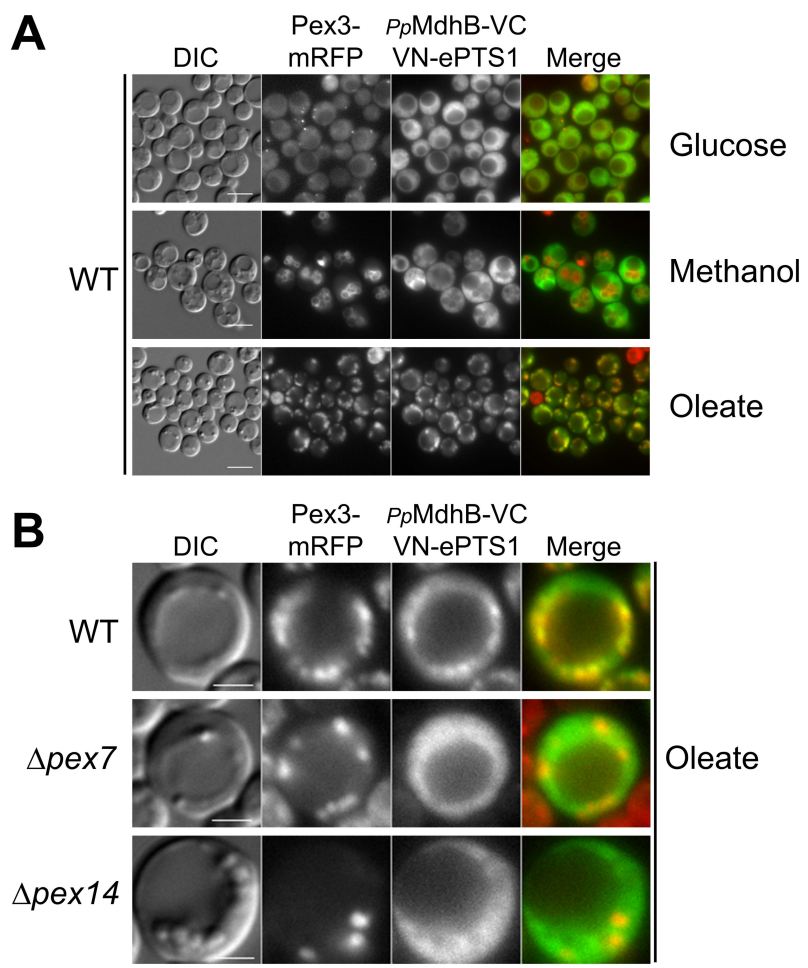

Figure 5. Localization of $P p \mathrm{MdhB}$ using the divergent BiFC assay. (A) Wild-type cells were grown overnight in glucose, methanol, and oleate. Bars: $5 \mu \mathrm{m}$. (B) Wild-type, $\Delta p e x 7$, and $\Delta p e x 14$ cells grown overnight in oleate. Bars: $2 \mu \mathrm{m}$. Peroxisomes were visualized using Pex3-mRFP.

In order to further study the peroxisomal import of $P p \mathrm{MdhB}$, we transformed $P p \mathrm{MdhB}$ VC and VN-ePTS1, as well as Pex3-mRFP, into $\Delta p e x 7$ and $\Delta$ pex14 cells. In oleate, similar to Pot1 in $\Delta p e x 7$ and $\Delta$ pex 14 cells, $P p$ MdhB displayed cytosolic fluorescence in $\Delta p e x 7$ cells, suggesting its peroxisomal import in a manner that is dependent on the PTS2 pathway (Figure 5B). As expected, no peroxisomal fluorescence was observed for $P p M d h B$ when import to this organelle was blocked using $\Delta$ pex 14 cells (Figure 5B).

\subsection{VC Fused to the N-Terminus of PpMdhB Is Not Imported to Peroxisomes}

To analyze whether $P p \mathrm{MdhB}$ may have a noncanonical PTS1 created by an unknown mechanism, such as ribosomal readthrough or mRNA splicing, we also fused VC to the N-terminus of $P p \mathrm{MdhB}$ (VC-PpMdhB) and transformed VC-PpMdhB and VN-ePTS1, as well as Pex3-mRFP, into wild-type cells. We found VC-P $p$ MdhB to be cytosolic in glucose, methanol, and oleate (Figure S3). The lack of peroxisomal localization of VC-P $p \mathrm{MdhB}$ 
suggests that no PTS1 was exposed, but also indicates that an N-terminal tag in PpMdhB hampers its interaction with the PTS receptor or carrier in wild-type cells.

\subsection{Independent Confirmation of the Peroxisomal Localization of PpMdhB-GFP in Oleate}

Because the peroxisomal fraction of PpMdhB may have eluded detection when it was expressed from its own promoter, we expressed $P p$ MdhB-GFP from the alcohol oxidase promoter $\left(\mathrm{P}_{\mathrm{AOX}}\right)$ that is tightly regulated, in an effort to reduce the cytosolic fluorescence and to make the peroxisomal fluorescence more apparent. $\mathrm{P}_{\mathrm{AOX}}$ is induced in the presence of methanol, strongly repressed by glucose, and not expressed in oleate. We transformed the plasmid containing P $p$ MdhB-GFP expressed from $\mathrm{P}_{\mathrm{AOX}}$ into wild-type cells, as well as plasmids expressing Tom20-mRFP and BFP-SKL as mitochondrial and peroxisomal markers, respectively, for colocalization studies.

As a control, we induced expression of $P p$ MdhB-GFP with methanol and found it to be fully cytosolic in cells grown overnight in methanol (Figure 6A). There was no colocalization with either peroxisomal or mitochondrial markers as expected. To control the levels of $P p \mathrm{MdhB}$, we turned on the expression of $P p \mathrm{MdhB}$ and peroxisome proliferation for only $2 \mathrm{~h}$ in methanol medium, turned off $P p \mathrm{MdhB}$ expression using glucose in the medium for $1 \mathrm{~h}$, and finally transferred the wild-type cells to oleate medium overnight for peroxisome proliferation (Figure $6 \mathrm{~B}$ ). Glucose should repress the AOX promoter and turn off peroxisome proliferation in methanol that usually results in clustered peroxisomes as observed in our control. With a limited production of $P p \mathrm{MdhB}$, we expected a portion of $P p \mathrm{MdhB}$ would remain in the cytosol, while another portion would localize to peroxisomes in oleate. Using this approach, we were indeed able to detect the peroxisomal isoform of $P p$ MdhB colocalizing with BFP-SKL (Figure 6B).

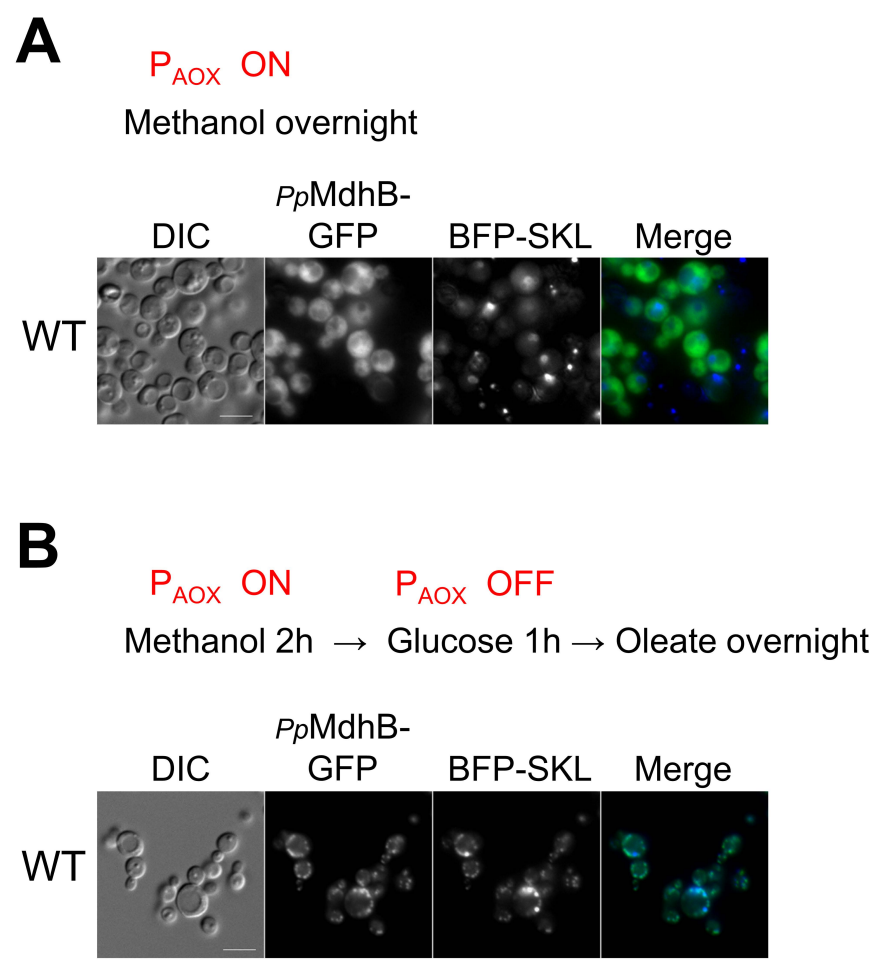

Figure 6. MdhB-GFP pulse-chase uncovers its localization to a small peroxisomal fraction. Fluorescence microscopy of $P p$ MdhB-GFP expressed from $P_{\text {AOX }}$. (A) Strains were grown overnight in methanol. (B) Strains were grown for $2 \mathrm{~h}$ in methanol to express PpMdhB-GFP ( $\left.\mathrm{P}_{\mathrm{AOX}} \mathrm{ON}\right)$, transferred to glucose for $1 \mathrm{~h}$ to repress the $A O X$ promoter $\left(\mathrm{P}_{\mathrm{AOX}} \mathrm{OFF}\right)$, and grown overnight in oleate to induce peroxisome proliferation. Colocalization studies with the peroxisome were observed with BFP-SKL as peroxisomal markers, respectively. Bars: $5 \mu \mathrm{m}$. 
These results show convincingly that the peroxisomal fluorescence of $P p \mathrm{MdhB}$, observed using the divergent $\mathrm{BiFC}$ assay, could be independently reproduced by controlling $P p$ MdhB-GFP expression levels resulting in peroxisomal $P p$ MdhB in oleate medium.

\section{Discussion}

While most proteins localized to the peroxisomal matrix have defined PTS sequences responsible for their localization to the peroxisome matrix, and the subcellular localization of such proteins is often conserved through evolution, determining the peroxisomal localization and its mechanisms has been challenging for a subset of peroxisomal matrix proteins. These may be divided into five broad categories-(1) those that have no obvious PTS, but might be transported into peroxisomes in a "piggyback" fashion in association with another protein with a bona fide PTS [14]; (2) proteins whose PTS is generated by unusual mechanisms, such as ribosomal readthrough past a canonical stop codon [15,16], alternative splicing [17-19] or the use of alternative transcription start sites [20] or translational start sites [21]; (3) proteins with dual localization, including peroxisomes [8], where the detection of a small peroxisomal pool may require techniques with high sensitivity [22]; (4) dynamic, environment-specific peroxisomal localization that might be missed by experimental analysis of the protein under the wrong environmental conditions [14,23-25]; and (5) post-translational activation or inhibition of a PTS [23,26].

The maintenance of the cellular redox balance between $\mathrm{NAD}^{+}$, and its reduced form $\mathrm{NADH}$, is critical for many metabolic processes, as exemplified by the fact that the oxidation and reduction of $\mathrm{NAD}^{+}$and NADH drive both catabolic and anabolic reactions [4]. Cellular $\mathrm{NAD}^{+}$levels are maintained by both de novo and salvage biosynthetic pathways [27]. The majority of NADH formation is connected to biomass production: Synthesis of proteins, nucleic acids, and highly reduced lipids [4]. In yeasts, since all fatty acid oxidation occurs in peroxisomes, this organelle is an important source of NADH when cells are grown in carbon sources, such as oleate. The peroxisomal $\beta$-oxidation of fatty acids consumes $\mathrm{NAD}^{+}$, yielding $\mathrm{NADH}$, which needs to shuttle out of the peroxisome to maintain a peroxisomal redox balance. Once NADH is in the cytosol, it shuttles to the mitochondria and is subsequently transferred to complex I in the electron transport chain, contributing to the production of ATP. NADH is also produced, albeit not intraperoxisomally, by methyltrophic yeasts grown in methanol, whose oxidation occurs by a process that reduces $\mathrm{NAD}^{+}$, yielding $2 \mathrm{NADH}$ in the cytosol, which shuttle to mitochondria for ATP production as well. Thus, we expected that some of the NADH-shuttling proteins in P. pastoris would be peroxisomally localized at least during growth in oleate, which produces intraperoxisomal $\mathrm{NADH}$, but we were surprised to find that this pool was not detectable by traditional subcellular localization methods.

We, therefore, developed a sensitive method to detect small pools of peroxisomallylocalized proteins. The method is based on a divergent use of the BiFC method, normally used to study interactions between proteins. It is based on the concept of protein crowding of two noninteracting proteins fused to $\mathrm{N}$ - and C-terminal halves of the fluorescent Venus protein, rather than their physical interaction, within a small subcellular compartment to generate a fluorescence signal. Such proximity, reflected by the presence of fluorescence, would then indicate that the two proteins must be colocalized. The divergent BiFC method used here directly visualizes the spatial and temporal interaction between two colocalized, noninteracting proteins in vivo. The fluorescence signal provides a sensitive readout for detecting protein colocalization even at a low expression level comparable to that of the endogenous proteins and detects peroxisomal localization even in the background of some cytosolic labeling.

After demonstrating a proof-of-concept with peroxisomally-localized Pot1-VC and VN-ePTS1 proteins, we used this divergent BiFC method to determine whether any of the NADH-shuttling proteins in P. pastoris were localized in peroxisomes. Our finding was that only $P p \mathrm{MdhB}$, but not $P p \mathrm{MdhA}$ or $P p \mathrm{Gpd} 1$, was partially peroxisomal and also cytosolic, but only in cells grown in oleate, and not in methanol or glucose. The localization of a 
protein to peroxisomes only in oleate is not unprecedented because, in S. cerevisiae, aspartate aminotransferase (Aat2) is cytosolic in glucose-grown cells, but peroxisomal in cells grown in oleate [28]. Indeed, recent studies show that several yeast proteins, particularly those involved in the $\beta$-oxidation of fatty acids, exhibit a priority for peroxisomal targeting in oleate, in comparison to glucose [29].

P $p$ MdhA-GFP and PpGpdA-GFP appeared to not only have partially cytosolic fluorescence, but also colocalized with the mitochondrial marker in glucose, methanol, and oleate media, consistent with the presence of high scoring mitochondrial targeting signals in these proteins. The organellar location of all three proteins was also confirmed independently using GFP fusions of these proteins, and particularly for $P p M d h B$ expressed in the pulse-chase experiment from the inducible $A O X$ promoter in methanol, and that was then turned off in oleate, thereby validating the divergent BiFC assay, as well as our conclusions.

\subsection{Physiological Roles of NADH-Shuttling Proteins in P. pastoris}

Our work suggests that PpGpdA may be the homolog of ScGpd2 based on its mitochondrial localization and may play a role in shuttling NADH from the cytosol to the mitochondria. In contrast to $S$. cerevisiae, P. pastoris does not appear to be shuttling NADH out of the peroxisome through the glycerol 3-phosphate shuttle, as peroxisomal fluorescence and colocalization with peroxisomal markers for $P p G p d A$ were not observed. P $p$ MdhA may be the homolog of ScMdh1 based on its mitochondrial localization and may play a role in the TCA cycle in the mitochondria, in addition to regenerating NADH in the mitochondria that were shuttled in from the cytosol through the malate shuttle. Overall, it appears that NADH may be shuttled from the cytosol to the mitochondria through the malate dehydrogenase shuttle and/or the glycerol-3 phosphate dehydrogenase shuttle in P. pastoris (Figure 7).

In addition to the two mitochondrial NADH shuttling homologs, based on its cytosolic localization in all three media, $P p \mathrm{MdhB}$ may be the homolog of $S c \mathrm{Mdh} 2$ by shuttling NADH generated in the cytosol from glucose and methanol metabolism, and NADH generated in peroxisomes from oleate metabolism, to the mitochondria and may play a role in gluconeogenesis and the glyoxylate cycle (Figure 7).

However, based on its peroxisomal localization in oleate, $P p \mathrm{MdhB}$ may also be the homolog of $S c \mathrm{Mdh} 3$ in playing a role in $\beta$-oxidation by re-oxidizing NADH generated from fatty acid $\beta$-oxidation back to $\mathrm{NAD}^{+}$to maintain the intraperoxisomal redox balance (Figure 7).

The peroxisomal and cytosolic locations of $P p \mathrm{MdhB}$ in oleate satisfies the metabolic requirement wherein $\mathrm{NADH}$ that is produced in the peroxisome can be shuttled out of the peroxisome for redox balance and energy production. In contrast, there is no physiological need for PpMdhB to be peroxisomal in methanol, wherein NADH is primarily produced in the cytosol (Figure 7). 

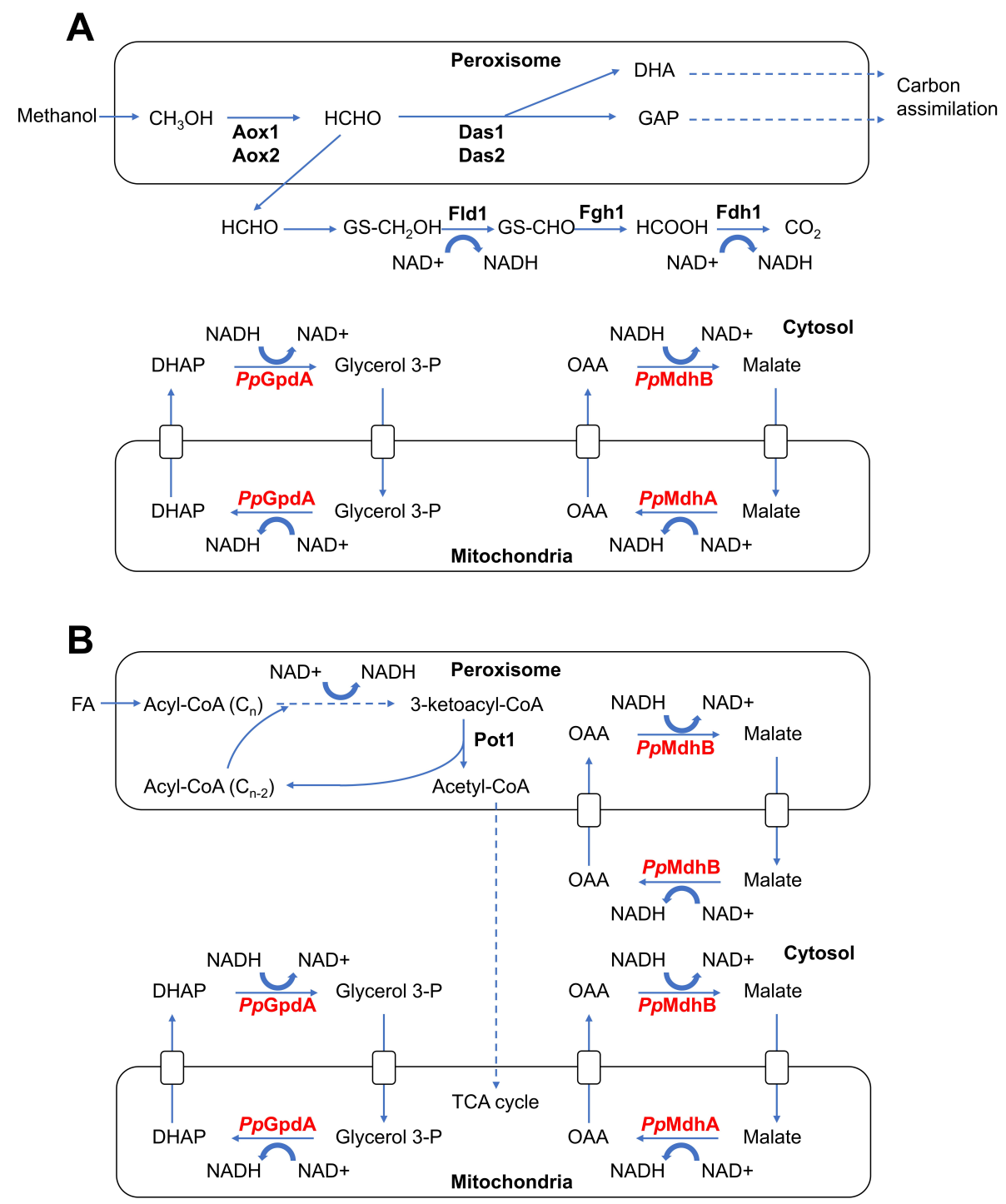

Figure 7. Proposed NADH shuttling in P. pastoris. (A) NADH shuttling in methanol. During methanol metabolism, methanol $\left(\mathrm{CH}_{3} \mathrm{OH}\right)$ is broken down into formaldehyde $(\mathrm{HCHO})$, producing hydrogen peroxide $\left(\mathrm{H}_{2} \mathrm{O}_{2}\right)$ as a byproduct. Some formaldehyde is converted in peroxisomes into dihydroxyacetone (DHA), and glyceraldehyde-3-phosphate (GAP) by dihydroxyacetone synthase 1 and 2 (Das1 and Das2), for carbon assimilation. Some formaldehyde diffuses into the cytosol, producing NADH through reactions involving Fld1 (formaldehyde dehydrogenase) and Fdh1 (formate dehydrogenase). $\mathrm{NADH}$ produced in the cytosol from methanol metabolism may be shuttled to the mitochondria for energy production through the malate or glycerol 3-phosphate (glycerol 3-P) shuttles. MdhB can convert oxaloacetate into malate, oxidizing $\mathrm{NADH}$ into $\mathrm{NAD}^{+}$. Malate enters the mitochondria through a transporter. MdhA converts malate back to oxaloacetate, reducing $\mathrm{NAD}^{+}$to NADH. GpdA can convert dihydroxyacetone phosphate (DHAP) into Glycerol 3-P, oxidizing NADH into $\mathrm{NAD}^{+}$. Glycerol 3-P can shuttle into the mitochondria where GpdA can regenerate NADH. (B) NADH shuttling in oleate. Fatty acid (FA), is actively transported into peroxisomes and acetylated to acyl-CoA in an ATP-dependent reaction. The newly acylated fatty acid is then desaturated, consuming FAD ${ }^{+}$and producing $\mathrm{H}_{2} \mathrm{O}_{2}$ as a byproduct. The newly formed, desaturated FA-CoA ester is hydrated, and then oxidized to form 3-ketoacyl-CoA and NADH. Finally, the 3-keto-acyl-CoA is cleaved by Pot1 (peroxisomal 3-oxyacyl-thiolase), releasing a molecule of acetyl-CoA and producing a fatty acyl-CoA, which is two carbons shorter than the substrate that entered the cycle. From this point, the product can restart the cycle. NADH produced in the peroxisomes during FA metabolism is shuttled out of the peroxisomes through the malate shuttle. MdhB converts oxalo-acetate into malate, regenerating $\mathrm{NAD}^{+}$. MdhB regenerates NADH in the cytosol and also shuttles $\mathrm{NADH}$ from the cytosol to the mitochondria. MdhB re-oxidizes $\mathrm{NADH}$ back to $\mathrm{NAD}^{+}$, reducing oxaloacetate to malate, which is transported into the mitochondria, where MdhA regenerates NADH in the mitochondria, converting malate back to oxaloacetate. The glycerol 3-P shuttle can also shuttle NADH from the cytosol to the mitochondria. 


\subsection{Targeting of $P p M d h B$ to Peroxisomes}

Although $P p \mathrm{MdhB}$ has a dual localization to the cytosol and peroxisome depending on the media, no recognizable PTS1 or PTS2 was found in $P p \mathrm{MdhB}$, despite our observation that the peroxisomal trafficking of $P p \mathrm{MdhB}$ requires a free $\mathrm{N}$-terminal end and depends on the PTS2 receptor, Pex7. Similar to P. pastoris, Y. lipolytica also has two MDH genes, $Y l M D H 1$ producing a mitochondrial isoform with a strong mitochondrial prediction based on MitoProt II and YlMDH2 encoding a varying ratio of cytosolic and peroxisomal isoforms (ending in a PTS1), due to alternative splicing depending on the media [17]. However, most $P$. pastoris genes do not have introns, only a single cDNA encoding $P p \mathrm{MdhB}$ has been reported and there are no splice donor, acceptor, or branch point sites located near the $5^{\prime}$ or $3^{\prime}$ ends of the coding region to suggest that $\mathrm{PpMdhB}$ could use alternative splicing to acquire a PTS2. The small fraction of peroxisomal $P p$ MdhB observed in the oleate medium may be due to one of the multiple mechanisms alluded to earlier in the Discussion. We also cannot rule out the presence of a novel or noncanonical PTS in PpMdhB that does not fit the known consensus for PTS1 or PTS2 sequences. There is precedent for noncanonical PTSs in several yeast proteins that use either the PTS1 receptor, Pex5 [30,31], or the PTS2 coreceptor, Pex20 [32], for entry into peroxisomes. Further work is necessary to determine which of the possible mechanisms is responsible for the peroxisomal targeting of $P p \mathrm{MdhB}$ in oleate.

\section{Materials and Methods}

\subsection{Yeast Strains and Media}

Strains used are shown in Supplementary Table S1. Growth medium used in this study: YPD ( $2 \%$ glucose, $2 \%$ bacto-peptone, $1 \%$ yeast extract), YNB $(0.17 \%$ yeast nitrogen base without amino acids and ammonium sulfate, $0.5 \%$ ammonium sulfate), CSM (complete synthetic medium of amino acids and supplements), glucose medium $(2 \times \mathrm{YNB}, 0.79 \mathrm{~g} / \mathrm{L}$ CSM, $0.04 \mathrm{mg} / \mathrm{L}$ biotin, $2 \%$ dextrose), oleate medium ( $2 \times \mathrm{YNB}, 0.79 \mathrm{~g} / \mathrm{L}$ CSM, $0.04 \mathrm{mg} / \mathrm{L}$ biotin, $0.02 \%$ Tween- $40,0.2 \%$ oleate), and methanol medium ( $2 \times$ YNB, $0.79 \mathrm{~g} / \mathrm{L} \mathrm{CSM}$, $0.04 \mathrm{mg} / \mathrm{L}$ biotin, $0.5 \%$ methanol).

\subsection{Plasmid Constructions}

Plasmids, shown in Supplementary Table S2, were constructed by Gibson Assembly. Gibson assembly primers were designed using NEBuilder version 2.5.2 (https: / / nebuilder. neb.com/\#!/ accessed on 14 March 2021). DNA was amplified from wild-type genomic DNA by PCR using Advantage 2 Polymerase (Cat\#639201; Takara Bio USA, Mountain View, CA, USA). Plasmid backbones were double-digested with the necessary restriction enzymes and purified using the Qiagen DNA purification kit (QIAGEN GmbH, Hilden, Germany). DNA inserts were cloned into the digested vectors using the NEBuilder Hifi DNA Assembly Mastermix (E2621S; New England Biolabs, Ipswich, MA, USA) and incubated for $1 \mathrm{~h}$ at $50{ }^{\circ} \mathrm{C}$. Newly-assembled plasmids were transformed into GC10 E. coli cells made chemically-competent. Then, $2 \mu \mathrm{L}$ of the Gibson Assembly mixture was added to the $50 \mu \mathrm{L}$ GC10 competent cells, placed on ice for $30 \mathrm{~min}$, heat shocked for $30 \mathrm{~s}$ at $42{ }^{\circ} \mathrm{C}$, placed on ice for $2 \mathrm{~min}$, and then $950 \mu \mathrm{L}$ of S.O.C. medium was added. The mixture was incubated at $37^{\circ} \mathrm{C}$ for $1 \mathrm{~h}$ shaking at $250 \mathrm{rpm}$ and then plated on LB plates with the appropriate antibiotic. Colonies were screened by colony PCR, and positive colonies were cultured in $5 \mathrm{~mL}$ of LB + antibiotic overnight. Plasmids were purified using the Wizard Miniprep Kit (Promega Corporation, Madison, WI, USA) and sequenced by Eton Bioscience.

\subsection{Yeast Strain Constructions}

Plasmids were linearized with the appropriate restriction enzyme before transforming into yeast cells made competent by electroporation. Yeast strains were cultured in a $250 \mathrm{~mL}$ flask with $50 \mathrm{~mL}$ of YPD shaking at $250 \mathrm{rpm}$ at $30{ }^{\circ} \mathrm{C}$ and grown until $1-2 \mathrm{OD}_{600} / \mathrm{mL}$ (exponential phase). Cells were pelleted by centrifugation at $3000 \mathrm{rpm}$ for $3 \mathrm{~min}$ and resuspended in $5 \mathrm{~mL}$ of YPD, $100 \mu \mathrm{L}$ of $1 \mathrm{M}$ HEPES (pH 8), and $125 \mu \mathrm{L}$ of $1 \mathrm{M}$ DTT. The 
resuspended cells were incubated at $30^{\circ} \mathrm{C}$ for $15 \mathrm{~min}$ on a lab rotator and then washed three times with cold, sterile water and once with $1 \mathrm{M}$ cold sorbitol. Cells were gently resuspended with 200-300 $\mu \mathrm{L}$ of cold sorbitol and placed on ice for $1 \mathrm{~h}$. Then, $50 \mu \mathrm{L}$ of cells and $5 \mu \mathrm{L}$ of linearized DNA were mixed together and transferred into a prechilled electroporation cuvette. After $10 \mathrm{~min}$ on ice, the mixture was electroporated using the BTX Harvard Apparatus Electroporator (BTX, San Diego, CA, USA) and was immediately resuspended in $1 \mathrm{M}$ cold sorbitol. The transformed cells were plated on YPD plates with the appropriate yeast selection markers and incubated at $30^{\circ} \mathrm{C}$ for a few days. Colonies were screened by Western Blot or fluorescence microscopy.

\title{
4.4. Fluorescence Microscopy
}

Cells were grown in YPD at $30{ }^{\circ} \mathrm{C}$ until exponential phase $\left(1-2 \mathrm{OD}_{600} / \mathrm{mL}\right)$, washed twice with sterile water, and then transferred to peroxisome proliferation media or used for microscopy. Cells were grown in oleate or methanol media overnight (16h) and washed twice with sterile water. Cells were then pelleted, $1.5 \mu \mathrm{L}$ of cells were mixed with $1 \%$ low melting point agarose and placed on a glass slide with a coverslip and imaged using $63 \times$ or $100 \times$ magnification on a Carl Zeiss Axioskop 2 MOT microscope (Carl Zeiss Microscopy, Gottingen, Germany). Images were taken on an AxioCam HRm digital camera(Carl Zeiss MicroImaging $\mathrm{GmbH}$, Gottingen, Germany); no digital gain was used, exposition for mitochondria or peroxisome markers was adjusted as needed, exposition for BiFC ranged between 200 to $1000 \mathrm{~ms}$ and was kept constant during microscopy in different media. Images were processed using AxioVision software V4.8.2.0 (Carl Zeiss Microscopy, White Plains, NY, USA). The images are representative results from experiments conducted at least in triplicate. Methodology to determine peroxisome or mitochondrial localization in different background strains is as follows: Cells showing distinct peroxisome labeling with Pex3-mRFP or BFP-SKL or mitochondrial labeling with Tom20-mRFP were first marked in the red or blue channel in the AxioVision software. Then, these marks were analyzed for colocalization in the green channel with the GFP-tagged proteins or fluorescence obtained from BiFC. No quantification was assessed or deemed necessary as we observed either total or no colocalization.

Supplementary Materials: Supplementary materials can be found online at https://www.mdpi. com/article/10.3390/ijms22094890/s1.

Author Contributions: Conceptualization, J.-C.F.; methodology, J.-C.F.; validation, J.-C.F. and P.L.; formal analysis, J.-C.F. and P.L.; Funding acquisition, S.S.; Investigation, P.L.; Supervision, J.-C.F. and S.S.; Writing-Original draft preparation, J.-C.F., P.L. and S.S.; Writing-Review \& editing, J.-C.F. and S.S. All authors have read and agreed to the published version of the manuscript.

Funding: This research was funded by the NIH grant (DK41737) to SS, who holds a Tata Chancellor's Endowed Professorship in Molecular Biology.

Data Availability Statement: No data other than those presented are applicable.

Acknowledgments: This work constitutes the BS/MS thesis of Paul Li.

Conflicts of Interest: The authors declare no conflict of interest.

\author{
Abbreviations \\ EYFP enhanced yellow fluorescent protein \\ BiFC Bimolecular Fluorescence Complementation \\ Mdh malate dehydrogenase \\ Gpd glycerol-3-phosphate dehydrogenase \\ PTS peroxisomal targeting signal \\ VN Venus N-terminal 1-158 amino acids \\ VC Venus C-terminal 159-239 amino acids
}




\section{References}

1. Hu, C.-D.; Chinenov, Y.; Kerppola, T.K. Visualization of interactions among bZIP and Rel family proteins in living cells using bimolecular fluorescence complementation. Mol. Cell 2002, 9, 789-798. [CrossRef]

2. Nagai, T.; Ibata, K.; Park, E.S.; Kubota, M.; Mikoshiba, K.; Miyawaki, A. A variant of yellow fluorescent protein with fast and efficient maturation for cell-biological applications. Nat. Biotechnol. 2002, 20, 87-90. [CrossRef]

3. Goodman, R.P.; Calvo, S.E.; Mootha, V.K. Spatiotemporal compartmentalization of hepatic NADH and NADPH metabolism. J. Biol. Chem. 2018, 293, 7508-7516. [CrossRef] [PubMed]

4. $\quad$ Bakker, B.M.; Overkamp, K.M.; van Maris, A.J.; Kotter, P.; Luttik, M.A.; van Dijken, J.P.; Pronk, J.T. Stoichiometry and compartmentation of NADH metabolism in Saccharomyces cerevisiae. FEMS Microbiol. Rev. 2001, 25, 15-37. [CrossRef]

5. Al-Saryi, N.A.; Al-Hejjaj, M.Y.; Van Roermund, C.W.T.; Hulmes, G.E.; Ekal, L.; Payton, C.; Wanders, R.J.A.; Hettema, E.H. Two NAD-linked redox shuttles maintain the peroxisomal redox balance in Saccharomyces cerevisiae. Sci. Rep. 2017, 7, 11868. [CrossRef] [PubMed]

6. Neuberger, G.; Maurer-Stroh, S.; Eisenhaber, B.; Hartig, A.; Eisenhaber, F. Prediction of peroxisomal targeting signal 1 containing proteins from amino acid sequence. J. Mol. Biol. 2003, 328, 581-592. [CrossRef]

7. Petriv, O.I.; Tang, L.; Titorenko, V.I.; Rachubinski, R.A. A new definition for the consensus sequence of the peroxisome targeting signal type 2. J. Mol. Biol. 2004, 341, 119-134. [CrossRef]

8. Rice, P.; Longden, I.; Bleasby, A. EMBOSS: The European Molecular Biology Open Software Suite. Trends Genet. 2000, 16, $276-277$. [CrossRef]

9. Claros, M.G.; Vincens, P. Computational method to predict mitochondrially imported proteins and their targeting sequences. J. Biol. Inorg. Chem. 1996, 241, 779-786. [CrossRef]

10. Shyu, Y.J.; Suarez, C.D.; Hu, C.D. Visualization of ternary complexes in living cells by using a BiFC-based FRET assay. Nat. Protoc. 2008, 3, 1693-1702. [CrossRef]

11. DeLoache, W.C.; Russ, Z.N.; Dueber, J.E. Towards repurposing the yeast peroxisome for compartmentalizing heterologous metabolic pathways. Nat. Commun. 2016, 7, 11152. [CrossRef] [PubMed]

12. Elgersma, Y.; Elgersma-Hooisma, M.; Wenzel, T.; McCaffery, J.M.; Farquhar, M.G.; Subramani, S. A mobile PTS2 receptor for peroxisomal protein import in Pichia pastoris. J. Cell Biol. 1998, 140, 807-820. [CrossRef] [PubMed]

13. Nazarko, T.Y.; Farré, J.-C.; Subramani, S. Peroxisome size provides insights into the function of autophagy-related proteins. Mol. Biol. Cell 2009, 20, 3828-3839. [CrossRef]

14. Al Saryi, N.A.; Hutchinson, J.D.; Al-Hejjaj, M.Y.; Sedelnikova, S.; Baker, P.; Hettema, E.H. Pnc1 piggy-back import into peroxisomes relies on Gpd1 homodimerisation. Sci. Rep. 2017, 7, 42579. [CrossRef] [PubMed]

15. Hofhuis, J.; Schueren, F.; Nötzel, C.; Lingner, T.; Gärtner, J.; Jahn, O.; Thoms, S. The functional readthrough extension of malate dehydrogenase reveals a modification of the genetic code. Open Biol. 2016, 6, 160246. [CrossRef] [PubMed]

16. Stiebler, A.C.; Freitag, J.; Schink, K.O.; Stehlik, T.; Tillmann, B.A.M.; Ast, J.; Bölker, M. Ribosomal readthrough at a short UGA stop codon context triggers dual localization of metabolic enzymes in fungi and animals. PLoS Genet. 2014, 10, e1004685. [CrossRef]

17. Kabran, P.; Rossignol, T.; Gaillardin, C.; Nicaud, J.-M.; Neuvéglise, C. Alternative splicing regulates targeting of malate dehydrogenase in Yarrowia lipolytica. DNA Res. 2012, 19, 231-244. [CrossRef]

18. Strijbis, K.; Burg, J.V.D.; Visser, W.F.; Berg, M.V.D.; Distel, B. Alternative splicing directs dual localization of Candida albicans 6-phosphogluconate dehydrogenase to cytosol and peroxisomes. FEMS Yeast Res. 2011, 12, 61-68. [CrossRef] [PubMed]

19. Freitag, J.; Ast, J.; Bölker, M. Cryptic peroxisomal targeting via alternative splicing and stop codon read-through in fungi. Nat. Cell Biol. 2012, 485, 522-525. [CrossRef] [PubMed]

20. Szewczyk, E.; Andrianopoulos, A.; Davis, M.A.; Hynes, M.J. A single gene produces mitochondrial, cytoplasmic, and peroxisomal NADP-dependent isocitrate dehydrogenase in Aspergillus nidulans. J. Biol. Chem. 2001, 276, 37722-37729. [CrossRef]

21. Wimmer, B.; Lottspeich, F.; Van Der Klei, I.; Veenhuis, M.; Gietl, C. The glyoxysomal and plastid molecular chaperones (70-kDa heat shock protein) of watermelon cotyledons are encoded by a single gene. Proc. Natl. Acad. Sci. USA 1997, 94, 13624-13629. [CrossRef] [PubMed]

22. Marelli, M.; Smith, J.J.; Jung, S.; Yi, E.; Nesvizhskii, A.I.; Christmas, R.H.; Saleem, R.A.; Tam, Y.Y.C.; Fagarasanu, A.; Goodlett, D.R.; et al. Quantitative mass spectrometry reveals a role for the GTPase Rho1p in actin organization on the peroxisome membrane. J. Cell Biol. 2004, 167, 1099-1112. [CrossRef] [PubMed]

23. Jung, S.; Marelli, M.; Rachubinski, R.A.; Goodlett, D.R.; Aitchison, J.D. Dynamic changes in the subcellular distribution of Gpd1p in response to cell stress. J. Biol. Chem. 2010, 285, 6739-6749. [CrossRef] [PubMed]

24. Kumar, S.; Singh, R.; Williams, C.P.; van der Klei, I.J. Stress exposure results in increased peroxisomal levels of yeast Pnc1 and Gpd1, which are imported via a piggy-backing mechanism. Biochim. Biophys. Acta 2016, 1863, 148-156. [CrossRef] [PubMed]

25. Valadi, Å.; Granath, K.; Gustafsson, L.; Adler, L. Distinct intracellular localization of Gpd1p and Gpd2p, the two yeast isoforms of NAD+-dependent glycerol-3-phosphate dehydrogenase, explains their different contributions to redox-driven glycerol production. J. Biol. Chem. 2004, 279, 39677-39685. [CrossRef]

26. Meyer, T.; Hölscher, C.; Schwöppe, C.; Von Schaewen, A. Alternative targeting of Arabidopsis plastidic glucose-6-phosphate dehydrogenase G6PD1 involves cysteine-dependent interaction with G6PD4 in the cytosol. Plant J. 2011, 66, 745-758. [CrossRef]

27. Lin, S.-J.; Guarente, L. Nicotinamide adenine dinucleotide, a metabolic regulator of transcription, longevity and disease. Curr. Opin. Cell Biol. 2003, 15, 241-246. [CrossRef] 
28. Verleur, N.; Elgersma, Y.; Roermund, C.W.T.; Tabak, H.F.; Wanders, R.J.A. Cytosolic aspartate aminotransferase encoded by the AAT2 gene is targeted to the peroxisomes in oleate-grown Saccharomyces cerevisiae. JBIC J. Biol. Inorg. Chem. 1997, 247, 972-980. [CrossRef]

29. Rosenthal, M.; Metzl-Raz, E.; Bürgi, J.; Yifrach, E.; Drwesh, L.; Fadel, A.; Peleg, Y.; Rapaport, D.; Wilmanns, M.; Barkai, N.; et al. Uncovering targeting priority to yeast peroxisomes using an in-cell competition assay. Proc. Natl. Acad. Sci. USA 2020, 117, 21432-21440. [CrossRef]

30. Rymer, Ł.; Kempiński, B.; Chełstowska, A.; Skoneczny, M. The budding yeast Pex5p receptor directs Fox $2 p$ and Cta1p into peroxisomes via its N-terminal region near the FxxxW domain. J. Cell Sci. 2018, 131, jcs216986. [CrossRef]

31. Kempinski, B.; Chelstowska, A.; Poznanski, J.; Krol, K.; Rymer, L.; Frydzinska, Z.; Girzalsky, W.; Skoneczna, A.; Erdmann, R.; Skoneczny, M. The peroxisomal targeting signal 3 (PTS3) of the budding yeast acyl-CoA oxidase is a signal patch. Front Cell Dev. Biol. 2020, 8, 198. [CrossRef] [PubMed]

32. Riccio, V.; Demers, N.; Hua, R.; Vissa, M.; Cheng, D.T.; Strilchuk, A.W.; Wang, Y.; McQuibban, G.A.; Kim, P.K. Deubiquitinating enzyme USP30 maintains basal peroxisome abundance by regulating pexophagy. J. Cell Biol. 2019, 218, 798-807. [CrossRef] [PubMed] 